\title{
Differential PARP cleavage:An indication for existence of multiple forms of cell death in human gliomas
}

\author{
Vasantha Kumar Bhaskara ${ }^{1,2}$, Sundaram Challa ${ }^{3}$, Manas Panigrahi ${ }^{3}$, Phanithi Prakash $\mathrm{Babu}^{1}$ \\ ${ }^{1}$ Department of Biotechnology, School of Life Sciences, University of Hyderabad, Hyderabad (AP) India, ${ }^{2}$ Stem Cell Laboratory, \\ Apollo Health Street, Apollo Hospitals Enterprise Ltd, Hyderabad (AP) India, ${ }^{3}$ Department of Pathology and Neurosurgery, NIMS, \\ Punjagutta, Hyderabad (AP), India.
}

\author{
Address for correspondence: \\ Prof. Phanithi Prakash Babu, \\ Department of Biotechnology, \\ School of Life Sciences, University of \\ Hyderabad, Hyderabad, India. \\ E-mail: ppbsl@uohyd.ernet.in
}

DOI: $10.4103 / 0028-3886.53265$

\begin{abstract}
Background: Gliomas represent a diverse range of clinical presentation, histological differentiation, and response to therapy. Altered cell proliferation and cell death signals in gliomas are of great interest to elucidate the key molecules involved and to find effective treatment modalities. By considering the role of different proteases in correlation with differential poly (ADP-ribose) polymerase (PARP) fragmentation we have studied the pattern of cell death in human glioma tissues. Materials and Methods: In our study, five different human glioma biopsies were collected and analyzed for the PARP cleavage pattern by using western immunoblotting. Samples were also analyzed for pro-caspase 3, calpain I $(\mu)$ and II $(\mathrm{m})$, granzyme-B and apoptosis-inducing factor (AIF). Parallel sections of histologically confirmed astrocytoma and glioblastoma multiforme (GBM) were used for immunohistochemical analysis of cleaved caspase-3, granzyme B, AIF and cyclo-oxygenase -2 (cox-2). Results: We found PARP fragmentation, along with usual $\sim 89 \mathrm{kDa}$ and $\sim 24 \mathrm{kDa}$ fragments, into other fragments of different molecular weights. Caspase mediated cell death may lead to appearance of larger $\sim 89 \mathrm{kDa}$ fragment and smaller $\sim 24 \mathrm{kDa}$ fragment indicating existence of apoptosis in the tumors. However, other fragments corresponding to $64 \mathrm{kDa}, \sim 54 \mathrm{kDa}$, and $\sim 40 \mathrm{kDa}$ were observed concomitantly in all glial tumor tissues. Conclusions: These results may indicate, not only apoptosis and necrosis, but there occurs the co-existence of intermediate cell death pathways in human glial tumors.
\end{abstract}

Key words: Caspase-3, human gliomas, multiple forms of cell death, poly (ADP-ribose) polymerase cleavage

\section{Introduction}

Glioblastoma multiforme (GBM) is the most common, aggressive and highly invasive form among the different glioma subtypes, which are characterized by the appearance of necrosis. ${ }^{[1]}$ Conventional thinking holds that a tumor develops necrosis when its growth rate outstrips its blood supply. However, pro-coagulation and anti-apoptotic mechanisms that result due to certain pathways could prevent completion of tumor necrosis factor (TNF)- $\alpha$ induced apoptosis instead it promotes the necrosis as an ultimate mode of cell death in a more complicated manner. ${ }^{[2]}$ In the n-ethyl n-nitrosourea (ENU)-induced glioma rat model it was reported that the tumor has an inherent property to adjust within the brain itself, by removing excess cells to accommodate newly forming cells. ${ }^{[3]}$ However, it was reported that there is no relation between cell death and cell proliferation. ${ }^{[4]}$ Cell death in glioma tumor tissues, is due to the existence of hypoxia that stimulates both apoptosis and necrosis. ${ }^{[5,6]}$ But the desired form of cell death is by apoptosis, which is inhibited in tumors. Moreover, the necrosis in tumors has an overall negative impact on the patient outcome. ${ }^{[7]}$ It was reported that hypoxia will provide a physiological selection in solid tumors for the expansion of variants that have lost their apoptotic potential. ${ }^{[8]}$ Increasing evidence shows that though cell death is indistinguishable by 
morphology they exhibit different signaling mechanism. ${ }^{[9]}$ Hypoxia-induced necrosis and distorted blood brain barrier in high-grade tumors are further responsible for development of peri-tumoral edema and associated complications. Hence, novel therapies directed to inhibit necrosis and to promote apoptosis have become significant in the treatment of gliomas.

Poly ADP-Ribose Polymerase (PARP) is a nuclear enzyme activated by DNA strand breaks and participates in DNA repair. PARP activity has a significant role in the rapid depletion of intra-cellular levels of adenosine tri-phosphate (ATP) and in shifting the cell death towards necrosis instead of apoptosis.$^{[10]}$ However, once the cell death cascade reaches the irreversible phase, PARP will be cleaved or inactivated. Thus the time period between rapid PARP activation, immediately after DNA damage and its cleavage has become crucial for the cells in selecting the mode of cell death. In the present study, we have correlated differential PARP fragmentation in human glioma biopsies with different protease activation. It was observed that elevated levels of different proteases correspond to the appearance of particular PARP fragment. This work may indicate that the cell death in glial tumors will coexist in multiple forms along with apoptosis and necrosis.

\section{Materials and Methods}

\section{Patient samples}

Five different glioma patient tumor samples were collected in the present study, as core tumor and peripheral tumor tissues. A part of the samples was used for tissue processing and the remaining samples were used for biochemical analysis. Informed consent was obtained from the patient/relatives before collecting the samples. Histopathological details of the samples used were elucidated earlier from our laboratory. ${ }^{[11]}$

\section{Immunohistochemistry}

Samples were fixed in $4 \%$ buffered paraformaldehyde and subsequently dehydrated in series of graded alcohol and then cleared by using chloroform. Finally, tissues were infiltrated and fixed in the paraffin to make tissue blocks. Thin sections of $6 \mu$ were taken and transferred onto pre-coated glass slides. Antigen unmasking was carried out by microwaving the sections in $10 \mathrm{mM}$ sodium citrate buffer $\mathrm{pH}$ 6.0. Sections were incubated with $3 \% \mathrm{H}_{2} \mathrm{O}_{2}$ (contained in $50 \%$ methanol) for 10-20 min. Blocking was done in 100 to $400 \mu \mathrm{l}$ of normal goat serum for 1-2 $\mathrm{h}$ at room temperature. Then sections were incubated along with the primary antibodies (polyclonal cleaved caspase- 3 obtained from cell signaling technology, Beverly, MA, USA; monoclonal granzyme B and polyclonal AIF obtained from oncogene research products, CA, USA: monoclonal cyclo-oxygenase-2 obtained from Genetix Biotech Asia pvt., ltd., New Delhi, India) overnight at $4^{\circ} \mathrm{C}$ in a humid chamber. Peroxidase conjugated secondary antibody was used for $1 \mathrm{~h}$ incubation time at room temperature followed by phosphate buffer saline (PBS) washes (3 X 5 min each). Again the sections were reincubated with $100-400 \mu$ lavidin-biotin complex (ABC) link for $30 \mathrm{~min}$ at room temperature. Sections were washed three times and incubated with 100-400 $\mu$ l of diaminobenzidine (DAB) chromagen solution till color develops. Hematoxylin was used as a counter stain. Finally, sections were dehydrated in series of graded alcohols and cleared in xylene. Mounting was done by using DPX and cover slips. All the reagents and secondary antibodies used in the protocol were obtained from DAKO Cytomation, California, USA.

\section{Western immunoblotting}

Samples were homogenized in the radio immunoprecipitation assay (RIPA) buffer, containing $50 \mathrm{mM}$ tris $\mathrm{HCl} \mathrm{pH} \mathrm{8.0,} 150 \mathrm{mM} \mathrm{NaCl}, 1 \mathrm{mM}$ EDTA, $0.4 \%$ deoxy cholate, $1 \%$ non-idet $\mathrm{p}-40$ containing protease inhibitor of $1 \mathrm{mM}$ PMSF and phosphatase inhibitors including $10 \mathrm{mM} \beta$-glycerophosphate, 10 $\mathrm{mM} \mathrm{NaF}, 0.3 \mathrm{mM} \mathrm{Na} 3 \mathrm{VO}$. Equal amounts of protein from different samples were separated on a SDS-PAGE. Further proteins were transferred onto a nitrocellulose membrane. Membranes were incubated for 12-14 h in primary antibodies (polyclonal anti-caspase-3, polyclonal full length anti-PARP obtained from cell signaling technology, Beverly, MA, USA: polyclonal anti-AIF, monoclonal anti-granzyme $B$ obtained from oncogene research products, CA, USA; monoclonal anti-calpain I and II obtained as a gift from Dr. Panaiyur S. Mohan, Nathan Kline Institute, NY, USA) at $4^{\circ} \mathrm{C}$. Blots were reincubated with the secondary antibodies conjugated to alkaline peroxidase (ALP) (anti-rabbit and anti-mouse IgG conjugated ALP obtained from Genei Pvt. Ltd., Bangalore, India; diluted 1:1000 in 5\% non-fat dry skim milk powder in TBS) for $1 \mathrm{~h}$ at room temperature. Immunoreactivity was visualized by incubating the blots with $33 \mu$ of 5-bromo 4-chloro-3-indoyl phosphate (BCIP) $5 \%$ and $66 \mu$ of nitro blue tetrazolium chloride monohydrate (NBT) $1 \%$ in $10 \mathrm{ml}$ of ALP buffer.

\section{Results}

Astrocytoma (WHO Grade II) and glioblastoma multiforme (WHO Grade IV) tissues were used in the present study after histopathological confirmation. Detailed clinical data is presented in Table 1.

Status of caspase- 3 among the glioma tissues

Caspase- 3 is one of the significant effector molecules 


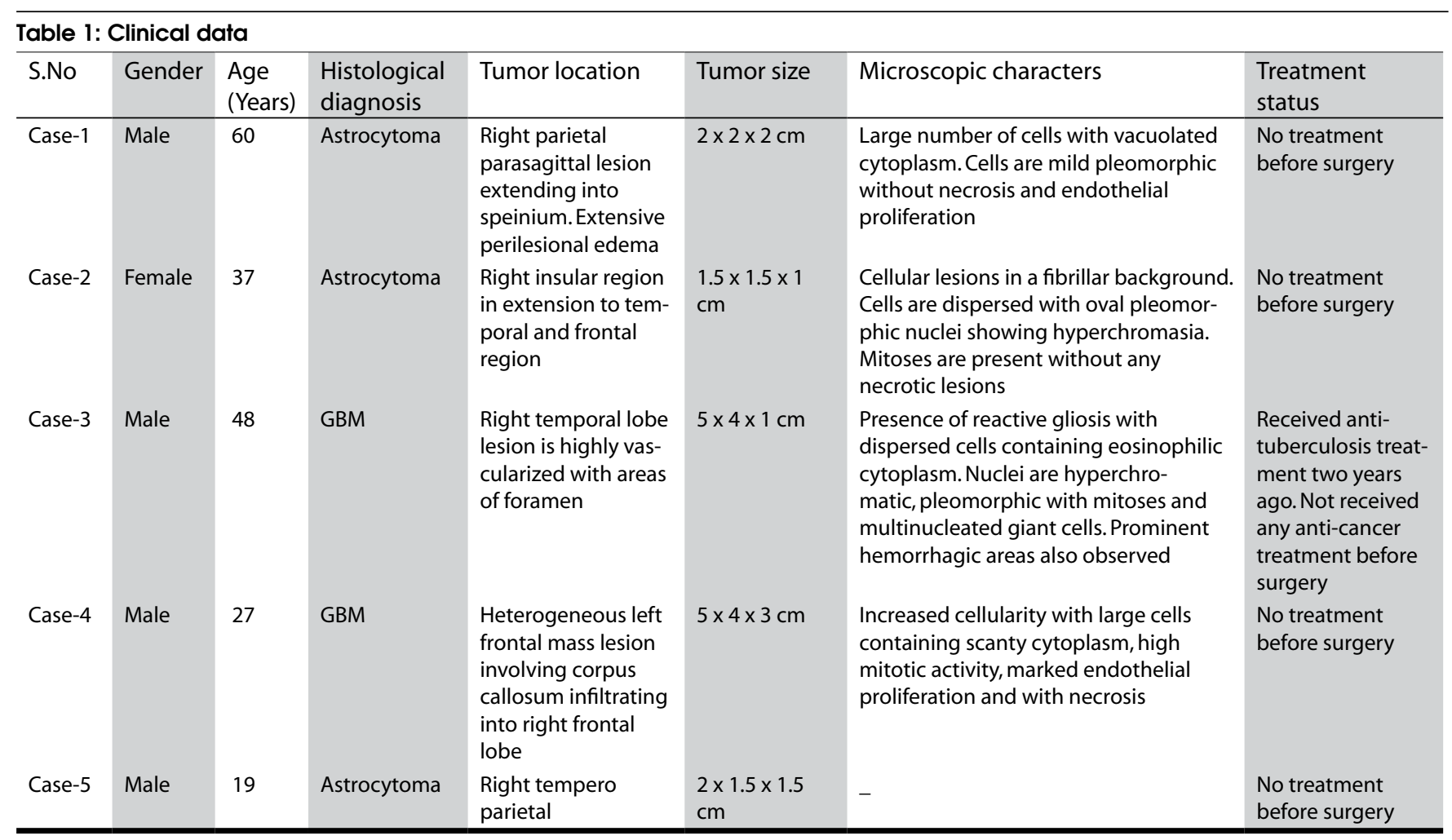

which is activated in both intrinsic and extrinsic forms of apoptosis. Procaspase-3 levels were found to be more in glioblastoma multiforme tumor samples, and almost absent in low-grade tumor samples (C2T and C5T) [Figure 1a]. However, immunohistochemical analysis of the tumor tissue sections by using cleaved caspase-3, clearly showed pronounced cytoplasmic and peri-nuclear positivity observed in the necrotic areas of glioblastoma multiforme [Figure $1 \mathrm{~b}$ and c]. Random distribution of mild positive cells was observed in astrocytoma tumor tissue sections [Figure 1d].

\section{Status of calpains, granzyme-B and PARP cleavage among the glioma tissues}

Calpains are cystein protease, present in the cytosol and get activated in relation to intracellular calcium levels. Elevated levels of smaller regulatory subunit $(30 \mathrm{kDa})$ of calpain I was found in core tumor tissues and at relatively higher levels in glioblastoma tumor (C4T) [Figure 2a]. Whereas higher levels of calpain II $(30 \mathrm{kDa})$ regulatory unit were found to be present in all the core tumor tissues compared to peripheral tissues [Figure 2b]. Granzyme-B is a serine protease secreted as a pre-proform and is further processed by lysosomal proteases to an active form, which acts on distinct substrates including caspases and PARP, thereby mediating apoptosis in the target cells by two different pathways. Granzyme-B levels were found to be higher in tumor core tissues and relatively more in glioblastoma [Figure 2c]. Granzyme-B immunohistochemistry has shown an increased cytoplasmic positivity in the cells present near the necrotic areas [Figure $2 b$ and e].

\section{AIF, Cox-2 and differential PARP cleavage pattern among the glioma tissues}

Apoptosis-inducing factor (AIF) is a mitochondrial protein that translocates into the nucleus and participates in caspase-independent form of cell death. Though immunoblot analysis showed increased levels of AIF nonuniformly among the samples, immunohistochemistry of AIF has shown increased nuclear positivity around the necrotic areas in glioblastoma sections [Figure 3a and b]. Cyclo-oxygenase-2 (Cox-2) function is well known to be an important mediator in inflammation. Cox-2 immunohistochemistry showed an increased cytoplasmic positivity in the cells present near the necrotic areas of glioblastoma [Figure $3 \mathrm{c}$ and $\mathrm{d}$ ]. Immunohistochemistry for granzyme-B, AIF, and Cox-2 among astrocytoma tissue sections have not shown any significant positive staining. Elevated levels of different proteases in the tumor tissues, ultimately cleaved full length PARP into different molecular weight fragments [Figure 3e].

\section{Discussion}

Cell death in gliomas as an important therapeutic target has been exploited by many researchers. Understanding the exact mode of cell death and the specific signaling mechanism involved in gliomas is highly significant 


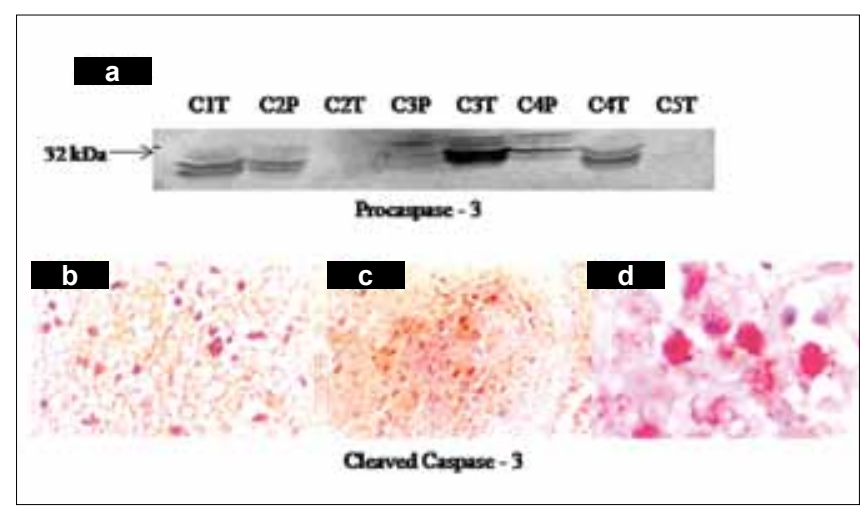

Figure 1: Pro-caspase-3 and activated or cleaved caspase-3 status in human glioma biopsies. Analysis of pro-caspase-3 expression by immunoblotting of human glioma biopsies (a), cleaved caspase-3 immunohistochemistry showing random distribution of positivity in astrocytoma $(10 X)(b)$ and pronounced cytoplasmic positivity in the necrotic areas of GBM (4X) (c) and (100X) (d)

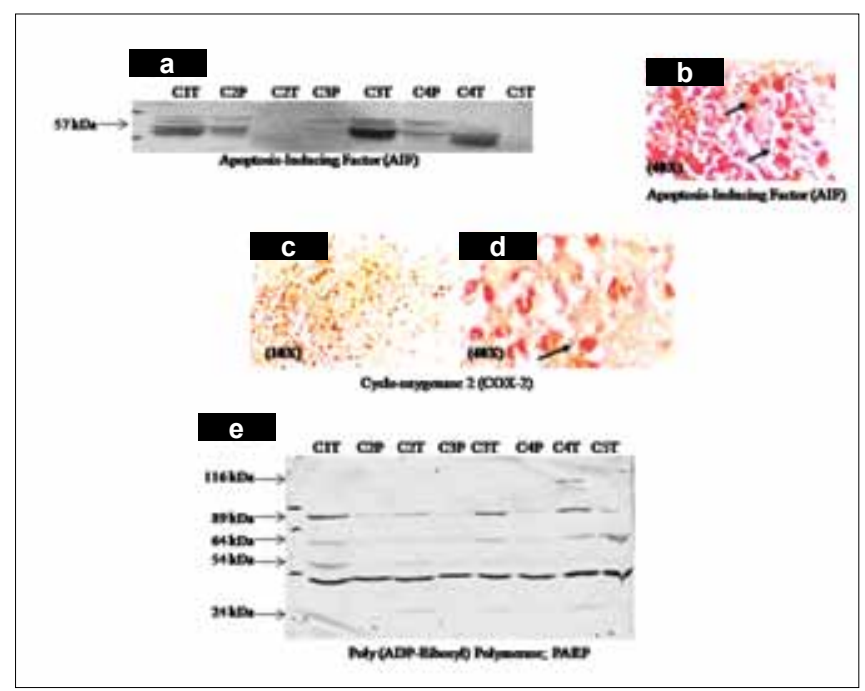

Figure 3: AIF status in human glioma biopsies. Tissue lysates showing AIF expression levels observed in the western immunoblot analysis (a), immunohistochemistry showing strong nuclear positivity in the areas of necrosis of glioblastoma tissues $100 \mathrm{X}$ (b), immunohistochemistry showing strong cytosolic positivity for Cox-2 in glioblastoma tissue sections 10X (c) and 100X (d), PARP)immunoblot showing multiple fragments in human gliomas (e)

for the successful induction of apoptosis and to inhibit unwanted form of cell death. Apoptosis and necrosis were reported to be the two major forms of cell death taking place in gliomas. However, the exact mode and mechanism of cell death remain unclear. Induction of hypoxic insult in rat fibroblastic cells leads to several distinct cell death programs based on the severity of the insult, which have intermediate features of both apoptosis and necrosis, termed as aponecrosis. ${ }^{[12]}$

In the present study a number of cells showing strong positivity for cleaved caspase-3 in GBM tumor samples

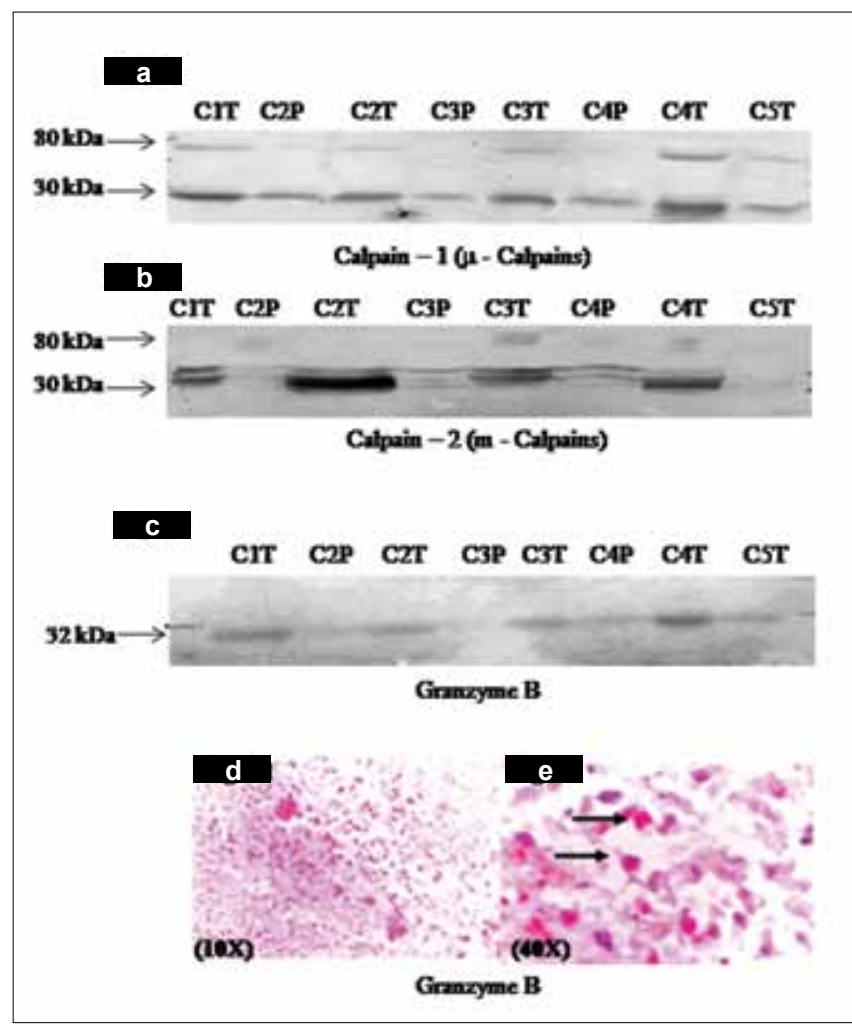

Figure 2: Calpains and granzyme-B status in human glioma biopsies. Analysis of \&\#61549;-calpain expression by immunoblotting of human gliomas (a), western immunoblot analysis of $\mathrm{m}$-calpain in human gliomas (b), tissue samples showing granzyme-B expression levels (c), immunohistochemistry showing strong cytoplasmic positivity in the cells present near the areas of necrosis in GBM 10X (d) and 40X (e)

around the areas of necrosis were observed. Consistently, earlier reports also indicated the presence of apoptotic cells in the glial tumors in the vicinity of the necrotic regions. ${ }^{[13]}$ Astrocytoma tumor sections showed mild positivity distributed randomly. We observed an increased level of smaller regulatory subunit $(30 \mathrm{kDa})$ of both calpain I and II. Higher levels were observed in the core tumor tissues compared to the peripheral tumor tissues. Increased m-calpain (calpain II) levels were observed in dystrophin-deficient muscle necrosis. ${ }^{[14]}$ Granzyme-B is a serine protease released from immune cells present in the tumor milieu in an inactive form, which is activated by lysosomal proteases of the target cells. Release of lysosomal cathepsin was reported in glial tumors. ${ }^{[15]}$ Increased granzyme-B levels of the tumors observed in the present study may correlate with the earlier reports demonstrating the increased cathepsin expression in brain tumors, may further indicate the existence of Type II (autophagy) cell death in gliomas. ${ }^{[16]}$ Thus elevated levels of cleaved caspase-3, calpain I (u) and II (m) and granzyme-B observed in the glioma tissues might have cleaved full length PARP ( 116kDa) into $89 \mathrm{kDa}$ and $24 \mathrm{kDa} ; 40 \mathrm{kDa} ; 64$ and $54 \mathrm{kDa}$ fragments respectively. Differential cleavage of 
PARP by different proteases into different fragments was reported earlier. ${ }^{[17]}$ AIF is another pro-apoptotic molecule, which can translocate from the mitochondria into the nucleus. In the nucleus, AIF will bind to the DNA and lead to large-scale DNA fragmentation and chromatin condensation. AIF can thus induce cell death in a caspase-independent manner. ${ }^{[18]}$ Increased nuclear positivity for AIF was observed in GBM tissues around the areas of necrosis. Western immunoblot for AIF appeared as multiple fragments around the $57 \mathrm{kDa}$ molecular weight. Cross-talk between the increased activity of PARP and AIF was known to initiate the nuclear signals that propagate mitochondria and further trigger the release of AIF leading to mitochondrial cell death. ${ }^{[19]}$ Cox-2 is an inducible enzyme, which can get rapidly activated in response to inflammation. We observed relatively more number of cells showing strong cytoplasmic positivity for Cox-2 in the areas of necrosis in GBM tissue sections. Enhanced expression of Cox-2 was reported in the breast, colon, prostate and lung carcinomas, whereas weak expression in normal colon epithelium. ${ }^{[20]}$ Hence, the present study may indicate the existence of intermediate forms of cell death along with well-known apoptosis and necrosis in human gliomas. However, in vitro experimentation using the glioma cell lines, to check the protease-specific PARP cleavage in response to a particular signal is yet to be confirmed.

\section{Acknowledgments}

The authors would like to acknowledge University Grants Commission (UGC), ICMR and ILS, for providing financial assistance.

\section{References}

1. Doolittle ND. State of the science in brain tumor classification. Semin Oncol Nurs 2004;20:224-30.

2. Raza SM, Lang FF, Aggarwal BB, Fuller GN, Wildrick DM, Sawaya R. Necrosis and glioblastoma:a friend or a foe? A review and a hypothesis. Neurosurgery 2002;51:2-12.

3. Nakajima M, Nakasu S, Morikawa S, Inubushi T. Estimation of volume doubling time and cell loss in an experimental rat glioma model in vivo. Acta Neurochir (Wien) 1998;140:607-12.

4. Schiffer D, Cavalla P, Chio A, Giordiana MT, Marino S, Mauro A, et al. Tumor cell proliferation and apoptosis in medulloblastoma. Acta
Neuropathol (Berl) 1994;87:362-70.

5. Tohma Y, Gratas C, Van Meir EG, Desbaillets I, Tenan M, Tachibana O, et al. Necrogenesis and Fas/APO-1 (CD95) expression in primary (de novo) and secondary glioblastoms. J Neuropathol Exp Neurol $1998 ; 57: 239-45$.

6. Kordeck R, Hironishi M, Liberski PP, Yanagihara R, Gajdusek DC. Apoptosis in glial tumors as determined by in situ non radioactive labeling of DNA breaks. Acta Neuropathol 1996;91:112-6.

7. Hammoud MA, Sawaya R, Shi W, Thall PF, Leeds NE. Prognostic significance of preoperative MRI scans in glioblastoma multiforme. J Neurooneol 1996;27:65-73.

8. Graeber TG, Osmanian C, Jacks T, Housman DE, Koch CJ, Lowe SW, et al. Hypoxia-mediated selection of cells with diminished apoptotic potential in solid tumours. Nature 1996;379:88-91.

9. Assuncao Guimaraes C, Linden R. Programmed cell deaths: Apoptosis and alternative deathstyles. Eur J Biochem 2004;271:1638-50.

10. Ha HC, Snyder SH. Poly (ADP-ribose) polymerase is a mediator of necrotic cell death by ATP depletion. Proc Natl Acad Sci USA 1999;96:13978-82.

11. Bhaskara VK, Manas P, Sundaram C, Babu PP. Comparative status of activated ERK1/2 and PARP cleavage in human gliomas. Neuropathology 2005;25:48-53.

12. Fomiglie L, Papucci L, Tani A, Schiavone N, Tempestini A, Orlandini GE, Capaccioli S, Orlandini SZ. Aponecrosis: morphological and biochemical exploration of a syncretic process of cell death sharing apoptosis and necrosis. J Cell Physiol 2000;182;41-9.

13. Steinbach JP, Wolburg H, Klumpp A, Probst H, Weller M. Hypoxia induced cell death in human malignant glioma cells: energy deprivation promotes decoupling of mitochondrial cyt c release from caspase processing and necrotic cell death. Cell Death Differ 2003;10:823-32.

14. Spencer MJ, Croall DE, Tidball JG. Calpains are activated in necrotic fibers from mdx dystrophic mice. J Biol Chem 1995;270:10909-14.

15. Sivaparvathi M, Sawaya R, Wang SW, Rayford A, Yamamoto M, Litta LA, et al. Overexpression and localization of cathepsin B during the progression of human gliomas. Clin Exp Metastasis 1995;13:49-56.

16. Gudinavicience I, Pranys D, Juozaityte E. Impact of morphology and biology on the prognosis of patients with gliomas. Midicina (Kaunas) 2004;40:112-20.

17. Shah GM, Shah RG, Poirier GG. Different cleavage pattern for poly (ADP-ribose) polymerase during necrosis and apoptosis in HL-60 cells. Biochem Biophys Res Commun 1996;229:838-44.

18. Joza N, Susin SA, Daugas E, Stanford WL, Cho SK, Li CY, et al. Essential role of the mitochondrial apoptosis-inducing factor in programmed cell death. Nature 2001;410;549-54.

19. Hong SJ, Dawson TM, Dawson VL. Nuclear and mitochondrial conversations in cell death:PARP-1 and AIF signaling. Trends Pharmocol Sci 2004;25:259-64.

20. Badie B, Schartner JM, Hagar AR, Prabakaran S, Peebles TR, Bartley B, et al. Microglia cyclooxygenase -2 activity in experimental gliomas: Possible role in cerebral edema formation. Clin Cancer Res 2003;9:872-7.

Accepted on 26-04-09

Source of Support: Nil, Conflict of Interest: None declared. 